\title{
Performance comparison of lithium-niobate-based extremely high-field single-cycle terahertz sources [Invited]
}

\author{
György Tóth", László Pálfalvi ${ }^{1}$, Szabolcs Turnár ${ }^{1}$, Zoltán Tibai ${ }^{1}$, Gábor Almási ${ }^{1,2}$, and János Hebling ${ }^{1,2,3^{*}}$ \\ ${ }^{1}$ Institute of Physics, University of Pécs, Pécs 7624, Hungary \\ ${ }^{2}$ Szentagothai Research Centre, University of Pécs, Pécs 7624, Hungary \\ 3 MTA-PTE High-Field Terahertz Research Group, Pécs 7624, Hungary
}

*Corresponding author: hebling@fizika.ttk.pte.hu

Received May 11, 2021 | Accepted July 9, 2021 | Posted Online August 30, 2021

\begin{abstract}
Tilted-pulse-front-pumping (TPFP) lithium-niobate terahertz (THz) pulse sources are widely used in pump-probe and control experiments since they can generate broadband THz pulses with tens of microjoules of energy. However, the conventional TPFP setup suffers from limitations, hindering the generation of THz pulses with peak electric field strength over $1 \mathrm{MV} / \mathrm{cm}$. Recently, a few setups were suggested to mitigate or even eliminate these limitations. In this paper, we shortly review the setups that are suitable for the generation of single-cycle THz pulses with up to a few tens of megavolts/centimeter focused electric field strength. The THz pulses available with the new layouts pave the way for previously unattainable applications that require extremely high electric field strength and pulse energy in the multi-millijoule range.
\end{abstract}

Keywords: terahertz pulse generation; nonlinear optics; ultrafast optics.

DOI: 10.3788/COL202119.111902

\section{Introduction}

Nonlinear terahertz $(\mathrm{THz})$ science started about two decades ago, when high-field $\mathrm{THz}$ pulses with frequencies of several tens of $\mathrm{THz}$ were generated in a GaSe semiconductor and were used as a pump pulse in pump-probe measurements ${ }^{[1]}$. THz pumpprobe measurement on the sub-THz to few- $\mathrm{THz}$ range became first possible, to the best of our knowledge, with the use of a lithium-niobate (LN) $\mathrm{THz}$ source pumped by near-IR ultrashort laser pulses in $2010^{[2]}$. Since $\mathrm{LN}$ has more than two times higher index of refraction in the $\mathrm{THz}$ range than in the near-IR range, tilted-pulse-front pumping (TPFP) is needed in order to achieve velocity matching for the optical rectification process in $\mathrm{LN} \mathrm{THz}$ sources $^{[3]}$. The velocity matching condition reads as

$$
v_{v}^{\mathrm{gr}} \cdot \cos \gamma=v_{\mathrm{THz}}^{\mathrm{ph}}, \quad \text { or } \quad n_{\mathrm{THz}}^{\mathrm{ph}} \cos \gamma=n_{p}^{\mathrm{gr}}
$$

in the TPFP THz pulse generation setups, where $v_{p}^{\mathrm{gr}}$ and $n_{p}^{\mathrm{gr}}$ are the group velocity and group index of refraction of the pump pulse, $v_{\mathrm{THz}}^{\mathrm{ph}}$ and $n_{\mathrm{THz}}^{\mathrm{ph}}$ are the phase velocity and (phase) index of refraction of the $\mathrm{THz}$ pulse, respectively, and $\gamma$ is the necessary pulse-front-tilt angle, which is $63^{\circ}$ for LN. The conventional TPFP THz source [see Fig. 1(a)] contains three main optical elements: (1) an optical grating to introduce a pulse front tilt, (2) a lens or an optical telescope to image the tilted pulse into the LN nonlinear optical material (NM), and (3) an LN crystal. The LN has to be prism-shaped with a wedge angle of $\gamma$ in order to make possible perpendicular in-coupling of the pump laser and outcoupling of the generated $\mathrm{THz}$ beam. (The $\mathrm{THz}$ beam generated inside the LN propagates at an angle $\gamma$ with respect to the propagation direction of the pump beam.) This conventional TPFP $\mathrm{LN} \mathrm{THz} \mathrm{source} \mathrm{has} \mathrm{become} \mathrm{very} \mathrm{popular} \mathrm{and} \mathrm{widely} \mathrm{used} \mathrm{in}$ THz pump-probe measurements and in experiments controlling material excitations ${ }^{[4]}$. In these cases, the typical $\mathrm{THz}$ pulse energy, focused $\mathrm{THz}$ field strength, and pump spot size are of a few microjoules $(\mu \mathrm{J})$, a few hundreds of kilovolts $(\mathrm{kV}) /$ centimeters $(\mathrm{cm})$, and a few millimeters $(\mathrm{mm})$, respectively.

Many possible applications of $\mathrm{THz}$ pulses have been proposed $^{[5-11]}$ and demonstrated ${ }^{[12-19]}$, which need the application of $\mathrm{THz}$ pulses with significantly above $1 \mathrm{MV} / \mathrm{cm}$ peak electric field strength or would strongly benefit from it. These applications include enhancement of both the high-harmonic generation efficiency ${ }^{[5]}$ and the cut-off frequency ${ }^{[6]}$, orientation of molecules $^{[12,13]}$, electron ${ }^{[9,10,15,19]}$ and proton $^{[8]}$ acceleration or other manipulation ${ }^{[7,17,18]}$, and generation of very special carrier-envelope stable attosecond pulses ${ }^{[11]}$. In addition to the above indicated megavolts (MV)/cm range of electric field, some of the previously mentioned applications also require well controllable single- or few-cycle $\mathrm{THz}$ pulses. For example, 
(i) alignment and orientation of molecules ${ }^{[12,13]}$ and (ii) efficient particle acceleration and manipulation ${ }^{[9,10,14]}$ are types of such applications. (i) The degree of both field-free orientation and alignment induced by single-cycle $\mathrm{THz}$ pulses with $\mu \mathrm{J}$ energies in an OCS gas sample was calculated and demonstrated in Refs. $[12,13]$. However, very recently, the advantage of applying single-cycle $\mathrm{THz}$ pulse with $\mathrm{MV} / \mathrm{cm}$ level field strength was demonstrated ${ }^{[20]}$. Numerical calculation predicted maximal orientation degrees for a single-THz pulse with $8 \mathrm{MV} / \mathrm{cm}$ field strength. (ii) Using a waveguide structure, which was pumped by $\sim 30 \mu \mathrm{J} \mathrm{THz}$ pulse, $200 \mathrm{MV} / \mathrm{m}$ acceleration gradient with $\sim 70 \mathrm{keV}$ energy gain was demonstrated, and the final electron energy of million electron volt $(\mathrm{MeV})$ level was predicted by applying $\mathrm{THz}$ pulses with few-MV/cm peak electric field strength $^{[19]}$. Another promising alternative accelerator scheme is the THz-driven inverse free-electron laser ${ }^{[18]}$. In the first demonstration, $150 \mathrm{keV}$ electron energy modulation was achieved in a magnetic undulator using $\sim 1 \mu$ J single-cycle $\mathrm{THz}$ pulse. Based on the calculations, the energy gain and acceleration gradient scaled linearly with the field of the $\mathrm{THz}$ pulse. Thereby, there is room for 2-3 orders of magnitude increase of the energy gain by increasing the $\mathrm{THz}$ field. Because of these and other important application possibilities, there is a strong need for developing $\mathrm{THz}$ sources that produce pulses with an extremely high field, having a few tens of $\mathrm{MV} / \mathrm{cm}$ peak electric field strength ${ }^{[21]}$.

In recent years, disadvantageous properties of the conventional TPFP setup have been identified ${ }^{[21-26]}$. These result in the hindrance of simple upscaling of the generated $\mathrm{THz}$ energy and field strength by increasing the pumping energy and the pumped area. However, nowadays, a few proposed setups promising mitigation or even elimination of these limitations were numerically investigated ${ }^{[22,23,26-34]}$. Although, the papers proposing the new setups usually compare the given new setups with the conventional TPFP setup, a comprehensive comparison of the newly suggested TPFP setups both to each other and to the conventional TPFP setup is missing yet. The present paper aims to present such a comparison.

In Sections 2 and 3, we shortly describe the most critical limiting properties of the conventional TPFP setup and describe the five newly proposed setups for mitigating/eliminating these limitations. In Section 4, we compare the main properties and best application possibilities of these setups.

\section{Limitations of the Conventional TPFP Setup}

The conventional TPFP THz source is shown schematically in Fig. 1(a). It contains three main optical elements: (1) an optical grating to introduce a pulse front tilt, (2) a lens or optical telescope to image the tilted pulse into the LN NM, and (3) a prismshaped LN crystal with a wedge angle of $\gamma$. Please note that the $\gamma$ tilt angle inside the LN crystal is set in three steps. First, the grating introduces a tilt angle $\gamma_{g}$. Its tangent will be changed by the imaging system [e.g., the lens in the case of Fig. 1(a)] having $M$ magnification. With the pump beam entering from the air $\left(n_{\text {air }} \approx 1\right)$ into the LN prism having $n_{p}^{\text {gr }}$ group index, the tangent

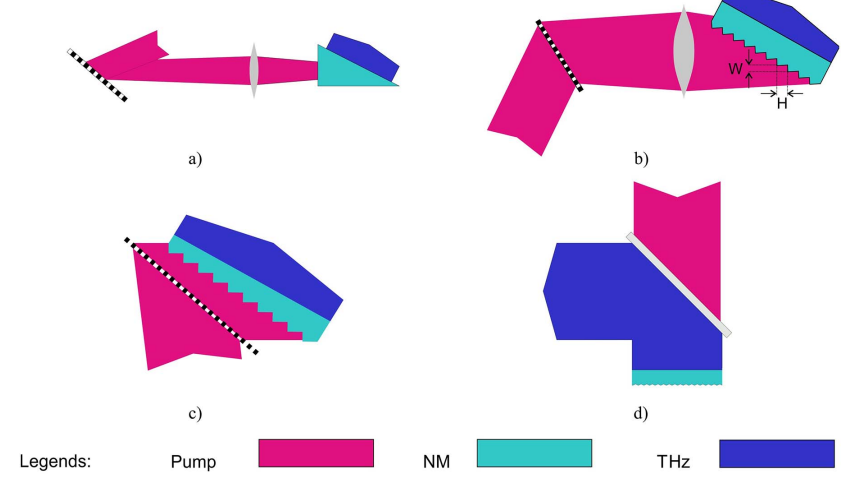

Fig. 1. Schematic depiction of the analyzed setups: (a) conventional TPFP (using optical grating) or TPFP setup with reflective echelon grating, (b) hybrid-contact-grating (with optical grating on NM) and NLES-I and echelon grating on NM, (c) NLES-wol setup, and (d) RNLS setup. See text for details.

of the tilt angle will be decreased by $n_{p}^{\text {gr }}$ times. So, the tangent of $\gamma$ is given as

$$
\tan \gamma=\frac{1}{n_{p}^{\mathrm{gr}} M} \tan \gamma_{g}
$$

Since for $\mathrm{LN} n_{\mathrm{THz}}^{\mathrm{ph}}$ is more than two times larger than $n_{p}^{\mathrm{gr}}$, according to Eq. (1), $\gamma$ has to be larger than $60^{\circ}$. The necessarily large tilt angle and the correspondingly large angular dispersion of the pump beam [see Eq. (3), where $n_{p}^{\mathrm{ph}}$ is the phase index of refraction of the LN at the $\lambda$ pump wavelength, and $\frac{\mathrm{d} \theta}{\mathrm{d} \lambda}$ is the angular dispersion of the pump beam ${ }^{[35]}$ result in three types of phenomena that cause serious difficulties or create limitations to increasing the peak electric field strength of the generated $\mathrm{THz}$ pulses:

$$
\tan \gamma=\frac{n_{p}^{\mathrm{ph}}}{n_{p}^{\mathrm{gr}}} \lambda \frac{\mathrm{d} \theta}{\mathrm{d} \lambda} .
$$

These limitations are the following:

(i) Imaging errors in the presence of angular dispersion result in significantly increased pump pulse duration at the edges of a large pump spot ${ }^{[26,27]}$. The longer pulse means reduced pump intensity and reduced local pumpto- $\mathrm{THz}$ conversion efficiency.

(ii) The prism shape of the LN crystal results in $\mathrm{THz}$ pulses with different temporal shapes across the $\mathrm{THz}$ beam cross section because of the different generation lengths. Such a bad quality, strongly asymmetric $\mathrm{THz}$ beam cannot be tightly focused, and thus high $\mathrm{THz}$ field strength cannot be achieved.

(iii) Due to the large group-delay dispersion (GDD) associated with the angular dispersion ${ }^{[35]}$, an ultrashort pump pulse evolves very fast inside the LN crystal. Consequently, the average pulse duration becomes much longer than the Fourier transform-limited (FL) pump pulse duration, resulting in strongly reduced effective 
$\mathrm{THz}$ generation length and pump-to- $\mathrm{THz}$ conversion efficiency ${ }^{[22]}$.

We notice that the negative effect of the limitation (iii) is less severe for longer FL pump pulses ${ }^{[22,36-38]}$ since, in this case, because of the smaller spectral bandwidth, the pump pulse evolves more slowly inside the LN.

\section{Setups with Reduced/Eliminated Limitations}

The following setups for TPFP LN THz sources with reduced or eliminated limitations have been proposed and numerically simulated.

\subsection{Hybrid-contact-grating setup}

In principle, limitations (i) and (ii) can be entirely eliminated with the application of the contact-grating setup ${ }^{[27-29,39]}$, which was proposed quite early ${ }^{[27]}$. An efficient contact-grating setup was realized using a $\mathrm{ZnTe}$ semiconductor as an NM, and as high as $0.3 \%$ pump-to $\mathrm{THz}$ energy conversion efficiency was demonstrated ${ }^{[0]}$. Contact-grating setups were designed and realized for LN too ${ }^{[28,29]}$. However, because of technical difficulties induced by the large necessary tilt angle (the need of a good quality contact grating with as high as about $3000 \mathrm{~mm}^{-1}$ groove density and the need to use an in-coupling prism ${ }^{[28]}$ ), efficient generation of $\mathrm{THz}$ pulses by using an LN contact-grating $\mathrm{THz}$ source has failed.

A new version of the contact-grating setup, the so-called hybrid-contact-grating setup, eliminates these difficulties. Here, the $\gamma$ tilt angle needed for velocity matching is introduced in two steps ${ }^{[26]}$. An optical-grating-based imaging system introduces a $\gamma_{\text {pre }} \approx \gamma$ pre-tilt, which without the effect of the contact grating would be reduced according to $\arctan \left[\left(\tan \gamma_{\text {pre }}\right) / n_{p}^{\mathrm{gr}}\right]$ when the pump pulse enters into the LN. As mentioned above in Eq. (2), such a reduction also happens in the case of the conventional TPFP setup. Because of this, in that case, the grating in the lens system has to introduce a $\gamma_{\text {air }}$ tilt angle according to $\tan \gamma_{\text {air }}=n_{p}^{\text {gr }} \tan \gamma$. However, in the hybrid-contact-grating setup, the diffraction of the pump beam on the contact grating at the entrance surface of the LN crystal introduces an additional tilt, ensuring the necessary tilt angle $\gamma$ inside the LN crystal. The substantial advantage of this setup compared with the original contact-grating setup ${ }^{[27]}$ is the reduced groove density of the contact grating from about 3000 to below $2000 \mathrm{~mm}^{-1}$. This reduction makes the preparation of the contact grating feasible in good quality. The other advantage is that there is no need for an in-coupling prism ${ }^{[26]}$. Although the $\mathrm{LN}$ still needs to be wedged, the wedge angle $\left(\leq 30^{\circ}\right)$ is less than half of that in the conventional TPFP setup. Furthermore, the imaging error becomes also significantly smaller, so larger pump spot size can be used.

\subsection{Setup with reflective echelon grating}

In order to eliminate limitation (iii), a $\mathrm{THz}$ setup that replaces the optical grating with an echelon grating in the conventional
TPFP setup [see Fig. 1(a)] has been proposed and investigated $^{[30]}$. Demonstrating the effective elimination of limitation (iii), $0.33 \%$ energy conversion efficiency was achieved with pumping by 70 -fs-long pulses. It is worth noting that although the pump intensity front inside the LN crystal is segmented (not continuous, it contains beamlets), the tilt angle of the average intensity front is the same as for the conventional TPFP setup. Consequently, the same angular dispersion could be present for long pump pulses ${ }^{[41]}$. However, for pump pulses shorter than the delay between the neighboring segments (beamlets) of the pump intensity front, the beamlets cannot interfere with each other, and the divergence of the whole beam is determined by the diffraction of the segment, which is smaller than the product of the angular dispersion [determined by Eq. (3)] and the spectral bandwidth of the pump pulse.

Since this setup contains a prism-shaped LN with the same wedge angle as the conventional TPFP setup, limitation (ii) is similarly effective in hindering using large pump spots and achieving extremely high $\mathrm{THz}$ energy.

\subsection{Nonlinear echelon slab with imaging}

This setup is depicted schematically in Fig. 1(b ${ }^{[31]}$. Similarly to the hybrid-contact-grating setup, this is also a hybrid setup. An optical-grating-based imaging system is used to introduce a pre-tilt angle of $\gamma$ in the air in front of the LN slab. An echelon structure with $W$ step width and $H$ step depth is manufactured into the originally plane parallel LN slab's front surface. Choosing an

$$
\frac{H}{W}=\tan \gamma
$$

ratio and tilting the LN slab by an angle of $\gamma$, the pump beam arrives perpendicularly to the steps, and the tilt angle of the envelope of the intensity front inside the $\mathrm{LN}$ is $\gamma$. The $\mathrm{THz}$ pulse generated by this pump intensity front will propagate perpendicularly to both the tangential planes of the front and back surfaces of the LN slab, and it will exit from the crystal without refraction. Inside the LN slab, the intensity front is segmented, but, contrary to the case of the reflection echelon grating setup, the intensity fronts in the beamlets are tilted, resulting in less significant "decoherence" (see Section 4.1) for the same $W$ step (beamlet) width. Since introducing $\gamma$ tilt angle in the air needs smaller angular dispersion than introducing the same $\gamma$ tilt inside LN, a smaller angular dispersion is present in the pump beam for the nonlinear echelon slab with imaging (NLES-I) setup than for the conventional setup. The smaller angular dispersion results in two disadvantages: both limitations (i) and (iii) become serious. A larger pump spot size can be used, leading to equal pulse elongation in the center and at the edges of the beam, and thus the effective $\mathrm{THz}$ generation length becomes much longer. 


\subsection{Nonlinear echelon slab without imaging}

This setup, depicted schematically in Fig. 1(c) ${ }^{[33]}$, differs from the NLES-I setup in two points. (1) In order to eliminate limitation (i), it does not contain an imaging element. Only a transmission grating is used to introduce the pulse front tilt. (2) Since the transmission grating is used in a symmetric (Littrow) arrangement to maximize its diffraction efficiency, a spatially varying chirp is present in the pump pulse after the diffraction. This effect is compensated by using wedged LN. The wedge angle is typically smaller than $10^{\circ}$. Different pre-tilt angles (typically in the range of $50^{\circ}-70^{\circ}$ ) can be used for different situations. An essential property of the setup is that for about $69^{\circ}$ pre-tilt angle and $9^{\circ} \mathrm{LN}$ wedge angle, the temporal shape of the THz pulses is almost perfectly independent of the transversal position. That is, despite the wedge shape of the LN crystal, spatially homogeneous $\mathrm{THz}$ pulses can be generated with a large beam size.

\subsection{Reflective nonlinear slab}

This setup, depicted schematically in Fig. $1(\mathrm{~d})^{[34]}$, is the simplest and most compact one. It has only a plane parallel LN slab with a back surface that is structured to form a blazed reflective grating or, for a rougher structure, a reflective echelon. The pump beam comes from the top of the figure. It practically does not generate any $\mathrm{THz}$ radiation when it propagates downward inside the NM since the velocity matching condition is not met. However, after it is diffracted or reflected on the structured backside, the velocity matching condition is fulfilled for the two partial beams (not shown in the figure), and $\mathrm{THz}$ radiation is generated.

Here, we mention that the type of the diffraction on the back surface depends on the relation between the $d$ period of the structure and the pump pulse duration. If the grating constant is large compared to the product of the pulse duration and the speed of light in LN, the individual parts of the beam cannot interfere with each other. In this case, the structure works as an echelon, and the tilted pulse front will be segmented. For this case, the reflective nonlinear slab (RNLS) works as a reflective nonlinear echelon slab (RNLES). In the opposite case, the structured back surface behaves like a special blazed reflection grating.

\section{Comparison of the New Setups}

$\mathrm{THz}$ generation is examined in detail from two aspects. On the one hand, the generation efficiency of the different setups is investigated at the typical pump wavelengths and pulse duration. On the other hand, the opportunity of the single-cycle $\mathrm{THz}$ pulse generation is analyzed. After that, the different setups are evaluated by several aspects.

\subsection{High-efficiency generation of $\mathrm{THz}$ pulses}

In the calculations, $\mathrm{Yb}$-doped yttrium aluminum garnet ( $\mathrm{Yb}$ : YAG) and Ti:sapphire lasers were assumed as pump lasers, as these types of ultrashort pulse lasers are available with high energy and high efficiency. For THz generation, the most critical differences between these lasers are the FL pulse duration and the wavelength. The shorter FL pulse duration changes faster during $\mathrm{THz}$ generation due to the large bandwidth in the presence of angular dispersion, and thereby the average pump intensity and generation efficiency will be significantly reduced. In the case of the shorter pumping wavelength $(800 \mathrm{~nm})$, three-photon absorption in LN becomes effective ${ }^{[42,43]}$. The free carriers generated by three-photon absorption of the pump result in significantly increased $\mathrm{THz}$ absorption. To avoid this effect, moderate pump intensity should be used, resulting in lower $\mathrm{THz}$ generation efficiency.

One-dimensional models were used to describe $\mathrm{THz}$ generation for the different setups ${ }^{[31,33,34]}$. These models calculate $\mathrm{THz}$ generation along the propagation direction of the generated $\mathrm{THz}$ pulse through the crystal. The effects of angular dispersion and linear dispersion due to the LN crystal were taken into account when the pump pulse duration was evaluated. The nonlinear optical effects on the pump pulse, such as the cascading effect and self-phase modulation ${ }^{[25]}$, were neglected. Since absorption by free carriers was also neglected, the results are optimistic in the case of $800 \mathrm{~nm}$ pumping wavelength. However, they reflect the relationships between the individual setups well.

In NLES and RNLES setups, the pump beam becomes segmented in the LN crystal due to the rough structured input and reflection surfaces of the LN slab, respectively. According to this fact, two significant effects were taken into account in the models. On the one hand, the "decoherence" effect was taken into consideration. By "decoherence," the following is meant: the $\mathrm{THz}$ wavelets generated at the different transversal points of the beamlets arrive with different phase delays at a distant point in the direction of propagation of the generated $\mathrm{THz}$ radiation. These phase differences can result in less constructive interference between the wavelets, causing a drop in the $\mathrm{THz}$ generation efficiency. On the other hand, the segmented parts of the beam can be considered in the model as independent beamlets ${ }^{[31,44]}$. These beamlets are diffracted during the propagation. The diffraction causes a decrease in the intensity of the beamlets, which also influences the generation efficiency negatively.

The dependencies of the efficiencies on the crystal length are presented in Fig. 2 in the case of the two different pump pulse sources for two different temperatures. The velocity matching frequency was supposed to be $0.5 \mathrm{THz}$ in all cases. In order to avoid the damage of the LN crystal and the unwanted nonlinear effects, the intensity was kept at $70 \mathrm{GW} / \mathrm{cm}^{2}$ and $140 \mathrm{GW} / \mathrm{cm}^{2}$ in the case of $200 \mathrm{fs}$ and $50 \mathrm{fs}$ pump pulse duration, respectively, similarly to Ref. [33].

According to the calculations, the conventional setup could achieve one of the highest efficiencies in the case of $200 \mathrm{fs} F$ pump pulses and room temperature operation [see Fig. 2(a)]. However, in this case, the usable beam size is very limited (approximately $1 \mathrm{~mm}$ ) because of both the decreasing generation efficiency and transversal inhomogeneity of the $\mathrm{THz}$ pulse 

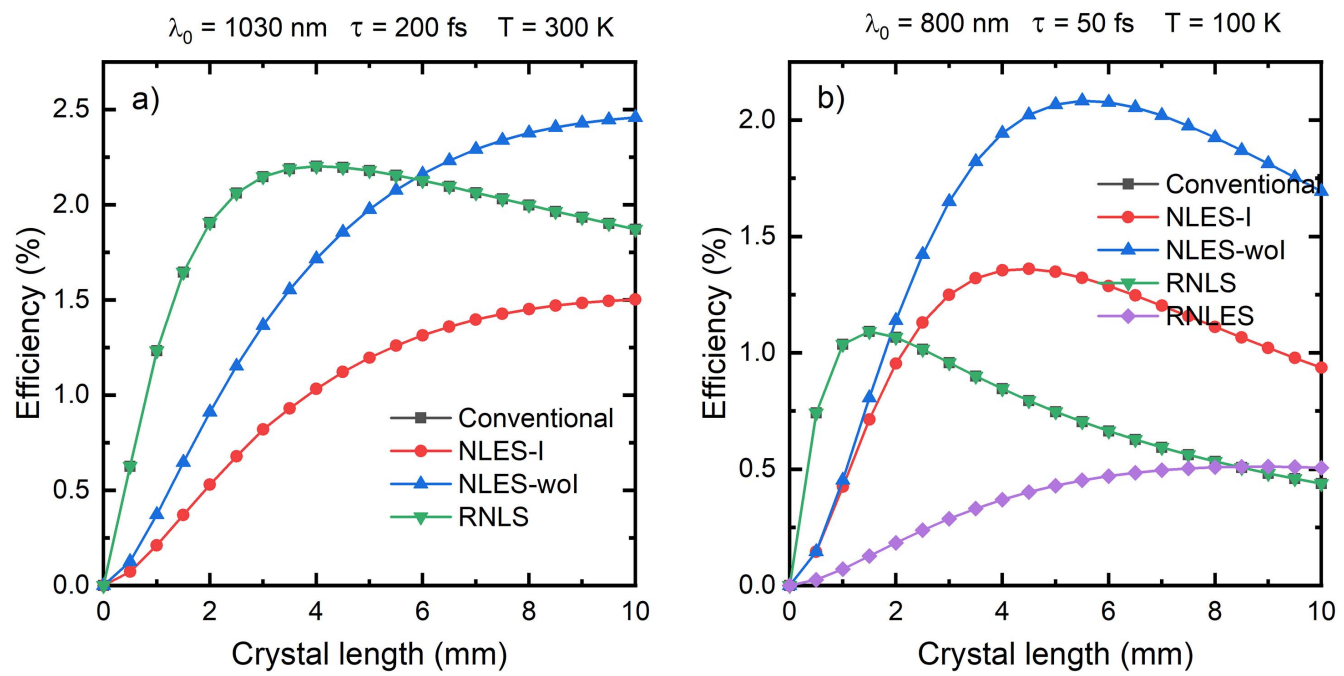

Fig. 2. Dependence of the efficiency on the crystal length using conventional, NLES-I, NLES-wol, and RNLS setups in case of two different pump pulse sources. In the case of an ultrashort pulse source, the RNLES setup was also investigated. The phase matching frequency was $0.5 \mathrm{THz}$.

shape with increasing pump beam size ${ }^{[33,45]}$. As it was mentioned above, the NLES-I setup could be used with a plane parallel LN slab to achieve perfect homogeneity. In this case, $1.5 \%$ $\mathrm{THz}$ generation efficiency could be approached for large crystal thickness. The beam waist radius of the pump can be $16 \mathrm{~mm}$ without significant imaging errors ${ }^{[31]}$. Assuming a pump pulse with a super-Gaussian spatial profile and $70 \mathrm{GW} / \mathrm{cm}^{2}$ peak intensity, the corresponding pump pulse energy is $90 \mathrm{~mJ}$. So, using this setup, the generated $\mathrm{THz}$ energy could be higher than $1 \mathrm{~mJ}$. Using the nonlinear echelon slab without imaging (NLESwoI) setup, a near-perfectly uniform $\mathrm{THz}$ beam could also be achieved if the wedge angle is close to $9^{\text {o[33] }}$. In this case, only the wedged shape of the crystal limits the beam size at such a large value as $50 \mathrm{~mm}$. With the predicted generation efficiency of more than $2 \%$, this allows generating $\mathrm{THz}$ pulses with $15 \mathrm{~mJ}$ energy by $750 \mathrm{~mJ}$ pump pulse energy. The achievable efficiency by RNLS is similar to the one of the conventional setup. However, in the case of RNLS, there is no limitation for the pumping beam size, and the generated $\mathrm{THz}$ energy will be limited only by the pump pulse energy.

In the case of the conventional and RNLS setups, for significantly shorter than 100 fs pump pulse duration, the optimal crystal length is shorter than $2 \mathrm{~mm}$ [see Fig. 2(b)]. This is the result of limitation (iii), as is explained in Section 2 . The negative effect of the shorter crystal length on the $\mathrm{THz}$ generation efficiency can be partly compensated by using cryogenic operation temperature. Since the angular dispersion is two times smaller in the NLES setups than in the conventional and RNLS setups, the reduction of the effective $\mathrm{THz}$ generation length with decreasing pump pulse duration is less significant. According to Fig. 2(b), the optimal crystal thickness is $4-5 \mathrm{~mm}$, and the $\mathrm{THz}$ generation efficiency is 1.3 and 2.0 times larger than for the conventional and RNLS setups. The advantage of RNLES (in the absence of angular dispersion, only the smaller effect of the material dispersion of the LN crystal influences the variation of the pump pulse duration inside the crystal) becomes effective in the case of ultrashort (less than 50 fs FL pulse duration) pump pulses.

\subsection{Single-cycle THz pulse generation}

For many applications, a strict single-cycle temporal shape of the $\mathrm{THz}$ pulses has outstanding importance. Therefore, the condition of single-cycle $\mathrm{THz}$ pulse generation was investigated for the different setups. Figures 3(a)-3(c) show the amplitude ratio of the side peak [see Fig. 4(a)] of the THz pulse to its mean peak as a function of the pump pulse duration and the crystal thickness. The corresponding $\mathrm{THz}$ generation efficiencies are shown in Figs. 3(d)-3(f). The shapes of the generated $\mathrm{THz}$ pulses and their spectra are shown in Fig. 4 for $E_{\text {side }} / E_{\max }=10 \%$.

According to Figs. 3(a)-3(c), the longer the pump pulse lengths are, the more single-pulse character the generated $\mathrm{THz}$ pulse has. The dispersion can explain this behavior in the $\mathrm{THz}$ spectral range. Really, it is expected that in the normal dispersion range the GDD results in such chirping of a traveling ultrashort pulse that the lower frequency appears on the leading part and the higher frequency on the trailing part, in agreement with Fig. 4. Using longer pump pulses, the generated $\mathrm{THz}$ frequencies are lower, and the effect of GDD is less significant. Of course, in the case of $\mathrm{THz}$ pulse generation in the LN crystal, other effects are also significant, and the dependence of the single-cycle character on the crystal length becomes more complex. For example, according to Fig. 3(a), in the case of NLES, the single-cycle character is almost independent of the crystal length.

So, for shorter than 500-fs-long pump pulses, the NLES setup is recommended for the generation of high-energy $\mathrm{THz}$ pulses with excellent beam quality. In the case of longer pump pulse durations, each presented setup is applicable for generating single-cycle $\mathrm{THz}$ pulses, but the simplest and most scalable is the $\mathrm{RNLS}^{[34]}$. It is worth mentioning that using intensity-modulated 

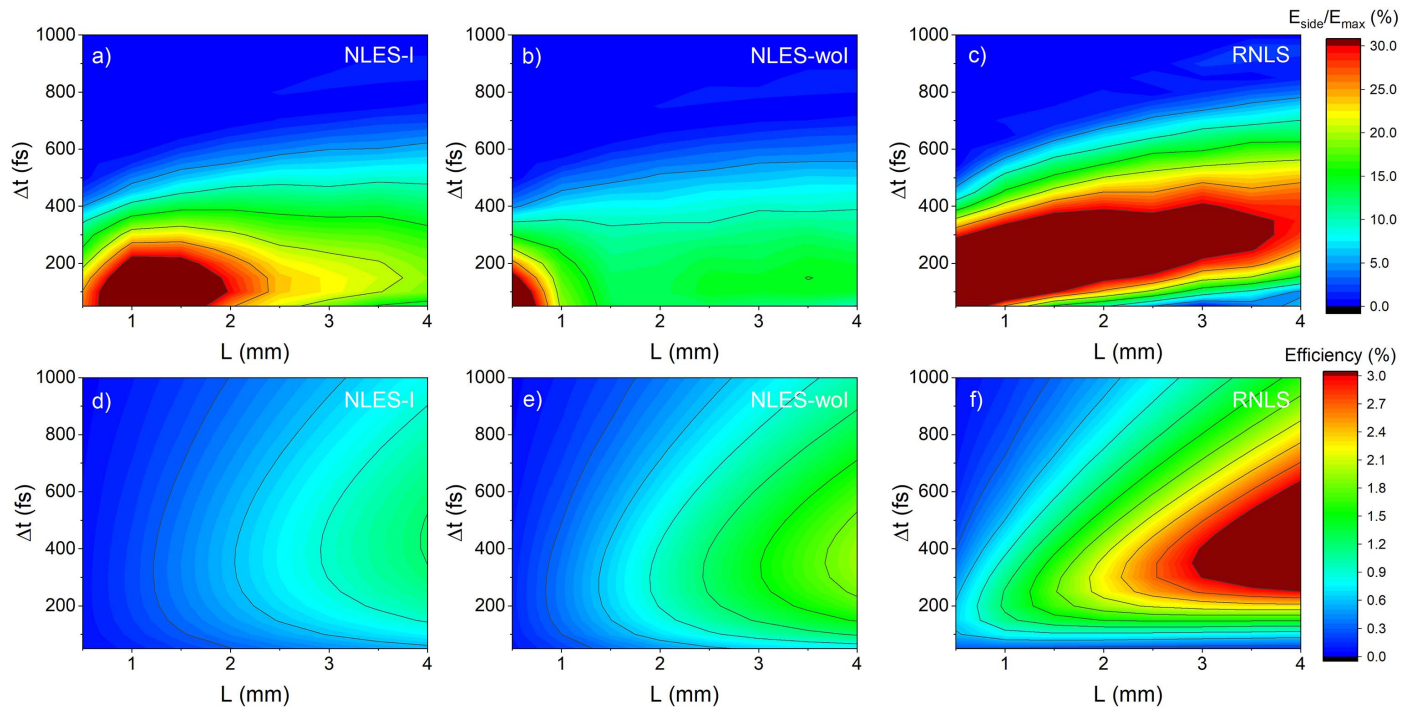

Fig. 3. (a)-(c) Ratio of the side peak to the main peak of the THz pulse and (d)-( $f$ ) the efficiency of the THz generation as a function of the pump pulse duration and the crystal thickness, for (a), (d) NLES-I, (b), (e) NLES-wol, and (c), (f) RNLS cases.
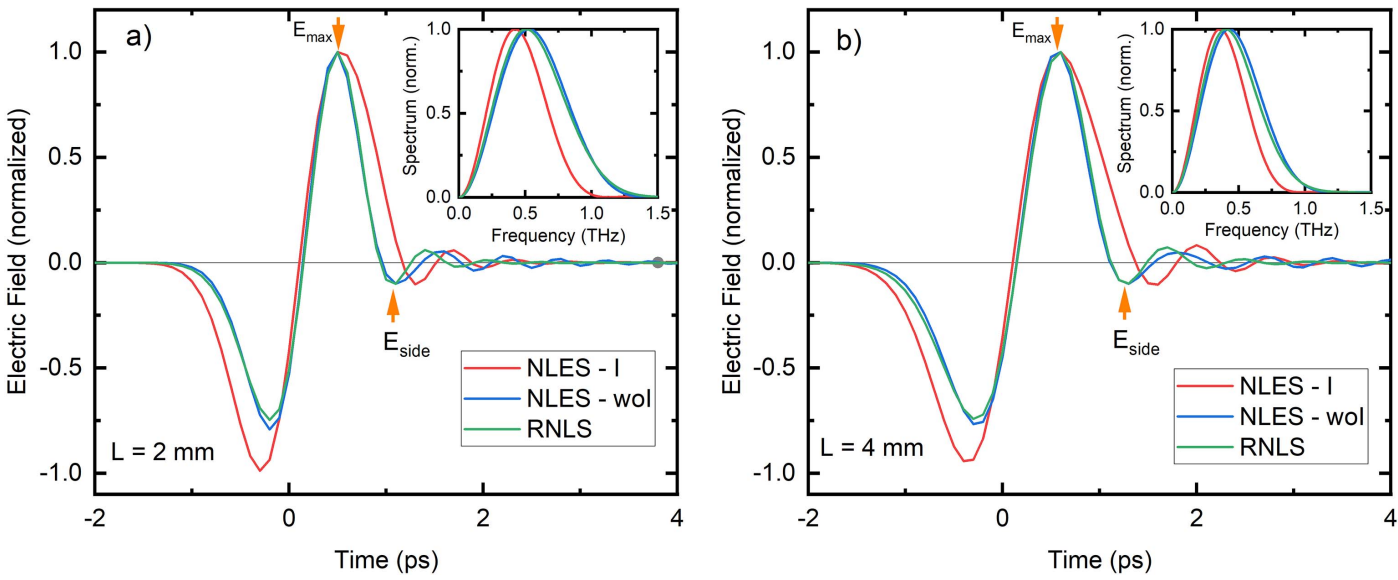

Fig. 4. Shapes and spectra (insets) of the generated THz pulses by NLES-I (red), NLES-wol (blue), and RNLS (green) setups for crystal lengths of (a) 2 mm and (b) $4 \mathrm{~mm}$. The pump pulse duration was appropriately chosen in order to satisfy the $E_{\text {side }} / E_{\max }=10 \%$ ratio. The corresponding pump pulse durations were (a)/ (b) $465 \mathrm{fs} / 475 \mathrm{fs}, 340 \mathrm{fs} / 390 \mathrm{fs}$, and $605 \mathrm{fs} / 700 \mathrm{fs}$ in the cases of NLES-I, NLES-wol, and RNLS, respectively.

pump pulses, all setups are suitable for generating multicycle $\mathrm{THz}$ pulses, too. Three important techniques were suggested to generate these kinds of pump pulses: chirp and delay technique $^{[46-48]}$, dual chirped optical parametric amplification technique $^{[49]}$, and technique based on the Vernier effect ${ }^{[50]}$.

\subsection{Recommendation}

Based on the discussion above and on our experience, we compared the most important properties of the different reviewed setups in Table 1. "Efficiency" means the pumped to $\mathrm{THz}$ conversion efficiency. "Scalability" means the energy scalability, which primarily depends on the useable pump beam size. "Beam quality" means the electric field homogeneity of the $\mathrm{THz}$ beam along the transversal dimension. "Shape stability" means how independent the waveform of the generated $\mathrm{THz}$ pulse is from the pump pulse length or the crystal length. "Feasibility" shows how easy (or difficult) it is to implement a given setup at the current state of the art.

It is important to note that every setup can work in echelon and optical-grating modes. In the case of echelon mode, the pulse front is discretely tilted, and it is segmented; in the case of conventional-grating mode, the pulse front tilt is continuous. The reflective echelon setups are the same as the conventional setup in the echelon mode. NLES-I is a special hybridcontact-grating setup in the echelon mode. The mode depends on the size of the grating groove relative to the spatial length of the pulse. If the grooves are not near enough to each other to interfere with the transmitted/reflected parts of the beam, the grating will work as an echelon grating, and the pulse front will be segmented. In the above discussion, including Table 1, it 
Table 1. Comparison of the Different THz Generation Setups ${ }^{a}$.

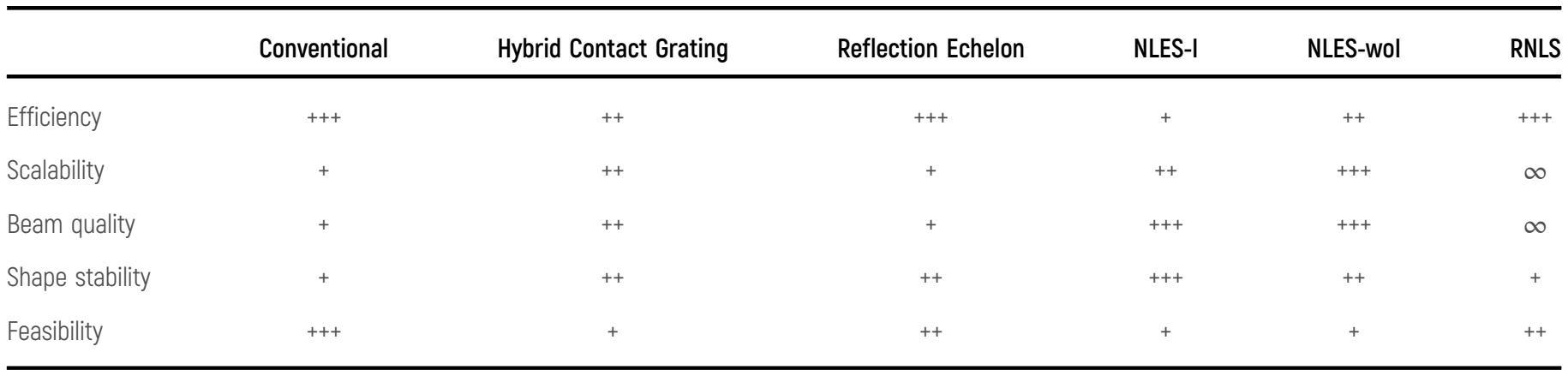

${ }^{a}$ More "+" signs mean better, and " $\infty$ " means theoretically perfect.

was assumed that the conventional, the hybrid contact grating, and the RNLS setups work in the optical-grating mode, while, the reflection echelon and both NLES setups work in the echelon mode. More sophisticated code ${ }^{[51]}$ was used to investigate a few of the setups presented here. That model predicts significantly smaller optimal crystal length and lower energy conversion efficiency than ours. However, it supports our main claim that the significant reduction (or even annulling) of the wedge angle of the crystal by the NLES technique results in higher conversion efficiencies and a much better pulse shape that is produced by the conventional setup. It is worth noting that if a smaller than Littrow incidence angle of the pump pulse is used on the grating in the NLES-woI setup, the wedge angle of the crystal could be further reduced ${ }^{[52]}$. Furthermore, according to our preliminary experiment ${ }^{[53]}$, it is possible to use a volume holographic grating (VHG) in the NLES-woI setup at a normal incidence angle without decreasing the diffraction efficiency. In this case, similarly to the NLES-I setup, a plane-parallel slab of LN can be used as NM, and our results obtained for the NLES-I setup become applicable.

\section{Conclusion}

The fundamental and practical limitations of recently proposed high-energy LN sources were analyzed. We have shown that the NLES-I, NLES-woI, and RNLS pumping schemes can significantly reduce the limitations of the conventional TPFP LN $\mathrm{THz}$ source. Performances of the different setups were compared, showing that a very significant enhancement of the available $\mathrm{THz}$ pulse energy and excellent beam quality can be expected by using the new setups compared to the conventional one. The only limiting factors are the available pump energy and crystal size in the case of RNLS. The RNLS setup will pave the way to new applications requiring multi-mJ $\mathrm{THz}$ pulses. Such critical applications are particle acceleration and manipulation, generation of attosecond pulses by high harmonic generation and other methods, orientation, and alignment of molecules, which all will gain from the availability of $\mathrm{THz}$ sources producing focused field strength in the few $10 \mathrm{MV} / \mathrm{cm}$ regime.

\section{Acknowledgement}

We thank Zsuzsanna Márton for the grammatical review of the manuscript. György Tóth thanks the János Bolyai Research Scholarship of the Hungarian Academy of Science for the support. This work has been supported by the National Research, Development and Innovation Office (125808, 129134); European Union, co-financed by the European Social Fund grant EFOP 3.6.2-16-2017-00005 entitled by Ultrafast physical processes in atoms, molecules, nanostructures, and biology structures. Project no. TKP2020-IKA-08 has been implemented with the support provided from the National Research, Development and Innovation Fund of Hungary, financed under the 2020-4.1.1-TKP2020 funding scheme. The UNKP-20-3 New National Excellence Program of the Ministry for Innovation and Technology from the source of the National Research, Development and Innovation Fund. Project no. 2018-1.2.1NKP-2018-00010 has been implemented with the support provided by the National Research, Development and Innovation Fund of Hungary, financed under the 2018-1.2.1-NKP funding scheme.

\section{References}

1. P. Gaal, W. Kuehn, K. Reimann, M. Woerner, T. Elsaesser, and R. Hey, "Internal motions of a quasiparticle governing its ultrafast nonlinear response," Nature 450, 1210 (2007).

2. J. Hebling, M. C. Hoffmann, H. Y. Hwang, K.-L. Yeh, and K. A. Nelson, "Observation of nonequilibrium carrier distribution in $\mathrm{Ge}, \mathrm{Si}$, and $\mathrm{GaAs}$ by terahertz pump-terahertz probe measurements," Phys. Rev. B 81, 035201 (2010).

3. J. Hebling, G. Almasi, I. Kozma, and J. Kuhl, "Velocity matching by pulse front tilting for large area THz-pulse generation," Opt. Express 10, 1161 (2010).

4. T. Kampfrath, A. Sell, G. Klatt, A. Pashkin, S. Mährlein, T. Dekorsy, M. Wolf, M. Fiebig, A. Leitenstorfer, and R. Huber, "Coherent terahertz control of antiferromagnetic spin waves," Nat. Photon. 5, 31 (2010).

5. E. Balogh, K. Kovacs, P. Dombi, J. A. Fulop, G. Farkas, J. Hebling, V. Tosa, and K. Varju, "Single attosecond pulse from terahertz-assisted high-order harmonic generation," Phys. Rev. A 84, 023806 (2011).

6. K. Kovacs, E. Balogh, J. Hebling, V. Tosa, and K. Varju, "Quasi-phase-matching high-harmonic radiation using chirped THz pulses,” Phys. Rev. Lett. 108, 193903 (2012). 
7. J. Hebling, J. A. Fulop, M. I. Mechler, L. Palfalvi, C. Toke, and G. Almasi, "Optical manipulation of relativistic electron beams using THz pulses," arXiv 1109.6852 (2011).

8. L. Palfalvi, J. A. Fulop, G. Toth, and J. Hebling, "Evanescent-wave proton postaccelerator driven by intense THz pulse," Phys. Rev. Accel. Beams 17, 031301 (2014).

9. Z. Tibai, M. Unferdorben, S. Turnar, A. Sharma, J. A. Fulop, G. Almasi, and J. Hebling, "Relativistic electron acceleration by focused THz pulses," J. Phys. B 51, 134004 (2018).

10. S. Turnár, J. Hebling, J. A. Fulop, G. Toth, G. Almasi, and Z. Tibai, "Design of a THz-driven compact relativistic electron source," J. Phys. B 127, 38 (2021).

11. G. Toth, Z. Tibai, A. Sharma, J. A. Fulop, and J. Hebling, "Single-cycle attosecond pulses by Thomson backscattering of terahertz pulses," J. Opt. Soc. Am. B 35, A103 (2018).

12. S. Fleischer, Y. Zhou, R. W. Field, and K. A. Nelson, "Molecular orientation and alignment by intense single-cycle THz pulses," Phys. Rev. Lett. 107, 163603 (2011).

13. K. N. Egodapitiya, S. Li, and R. R. Jones, "Terahertz-induced field-free orientation of rotationally excited molecules," Phys. Rev. Lett. 112, 103002 (2014).

14. W. R. Huang, E. A. Nanni, K. Ravi, K. H. Hong, A. Fallahi, L. J. Wong, P. D. Keathley, L. E. Zapata, and F. X. Kartner, "Toward a terahertz-driven electron gun," Sci. Rep. 5, 14899 (2015).

15. W. R. Huang, A. Fallahi, X. Wu, H. Cankaya, A.-L. Calendron, K. Ravi, D. Zhang, E. A. Nanni, K.-H. Hong, and F. X. Kartner, "Terahertz-driven, all-optical electron gun," Optica 3, 1209 (2016).

16. E. A. Nanni, W. R. Huang, K.-H. Hong, K. Ravi, A. Fallahi, G. Moriena, R. J. D. Miller, and F. X. Kartner, "Terahertz-driven linear electron acceleration," Nat. Commun. 6, 8486 (2015).

17. D. Zhang, A. Fallahi, M. Hemmer, X. Wu, M. Fakhari, Y. Hua, H. Cankaya, A.-L. Calendron, L. E. Zapata, N. H. Matlis, and F. X. Kartner, "Segmented terahertz electron accelerator and manipulator (STEAM)," Nat. Photon. 12, 336 (2018).

18. E. Curry, S. Fabbri, J. Maxson, P. Musumeci, and A. Gover, "Meter-scale terahertz-driven acceleration of a relativistic beam," Phys. Rev. Lett. 120, 094801 (2018).

19. D. Zhang, A. Fallahi, M. Hemmer, H. Ye, M. Fakhari, Y. Hua, H. Cankaya, A.-L. Calendron, L. E. Zapata, N. H. Matlis, and F. X. Kartner, "Femtosecond phase control in high-field terahertz-driven ultrafast electron sources," Optica 6, 872 (2019).

20. L. Xu, I. Tutunnikov, E. Gershnabel, Y. Prior, and I. S. Averbukh, "Long-lasting molecular orientation induced by a single terahertz pulse," Phys. Rev. Lett. 125, 013201 (2020).

21. P. Salen, M. Basini, S. Bonetti, J. Hebling, M. Krasilnikov, A. Y. Nikitin, G. Shamuilov, Z. Tibai, V. Zhaunerchyk, and V. Goryashko, "Matter manipulation with extreme terahertz light: progress in the enabling $\mathrm{THz}$ technology," Phys. Rep. 836-837, 1 (2019).

22. J. A. Fulop, L. Palfalvi, G. Almasi, and J. Hebling, "Design of high-energy terahertz sources based on optical rectification," Opt. Express 18, 12311 (2010).

23. M. Kunitski, M. Richter, M. D. Thomson, A. Vredenborg, J. Wu, T. Jahnke, M. Schoffler, H. S. Bocking, H. G. Roskos, and R. Dorne, "Optimization of single-cycle terahertz generation in $\mathrm{LiNbO}_{3}$ for sub-50 femtosecond pump pulses," Opt. Express 21, 6826 (2013).

24. K. Ravi, W. R. Huang, S. Carbajo, X. Wu, and F. X. Kartner, "Limitations to $\mathrm{THz}$ generation by optical rectification using tilted pulse fronts," Opt. Express 22, 20239 (2014).

25. S. B. Bodrov, A. A. Murzanev, Y. A. Sergeev, Y. A. Malkov, and A. N. Stepanov, "Terahertz generation by tilted-front laser pulses in weakly and strongly nonlinear regimes," Appl. Phys. Lett. 103, 251103 (2013).

26. L. Palfalvi, Z. Ollmann, L. Tokodi, and J. Hebling, "Hybrid tilted-pulse-front excitation scheme for efficient generation of high-energy terahertz pulses," Opt. Express 24, 8156 (2016).

27. L. Palfalvi, J. A. Fulop, G. Almasi, and J. Hebling, "Novel setups for extremely high power single-cycle terahertz pulse generation by optical rectification," Appl. Phys. Lett. 92, 171107 (2008).
28. Z. Ollmann, J. Hebling, and G. Almasi, "Design of a contact grating setup for mJ-energy THz pulse generation by optical rectification," Appl. Phys. B 108, 821 (2012).

29. M. Tsubouchi, K. Nagashima, F. Yoshida, Y. Ochi, and M. Maruyama, "Contact grating device with Fabry-Perot resonator for effective terahertz light generation," Opt. Lett. 39, 5439 (2014).

30. B. K. Ofori-Okai, P. Sivarajah, W. R. Huang, and K. A. Nelson, "THz generation using a reflective stair-step echelon," Opt. Express 24, 5057 (2016).

31. L. Palfalvi, G. Toth, L. Tokodi, Z. Marton, J. A. Fulop, G. Almasi, and J. Hebling, "Numerical investigation of a scalable setup for efficient terahertz generation using a segmented tilted-pulse-front excitation," Opt. Express 25, 29560 (2017).

32. P. S. Nugraha, G. Krizsan, C. Lombosi, L. Palfalvi, G. Toth, G. Almasi, J. A. Fulop, and J. Hebling, "Demonstration of a tilted-pulse-front pumped plane-parallel slab terahertz source," Opt. Lett. 44, 1023 (2019).

33. G. Toth, L. Palfalvi, J. A. Fulop, G. Krizsan, N. H. Matlis, G. Almasi, and J. Hebling, "Numerical investigation of imaging-free terahertz generation setup using segmented tilted-pulse-front excitation," Opt. Express 27, 7762 (2019).

34. G. Toth, L. Palfalvi, Z. Tibai, L. Tokodi, J. A. Fulop, Z. Marton, G. Almasi, and J. Hebling, "Single-cycle scalable terahertz pulse source in reflection geometry," Opt. Express 27, 30681 (2019).

35. J. Hebling, "Derivation of the pulse front tilt caused by angular dispersion," Opt. Quantum Electron. 28, 1759 (1996).

36. J. A. Fulop, L. Palfalvi, S. Klingebiel, G. Almasi, F. Krausz, S. Karsch, and J. Hebling, "Generation of sub-mJ terahertz pulses by optical rectification," Opt. Lett. 37, 557 (2012).

37. J. A. Fülöp, Z. Ollmann, C. Lombosi, C. Skrobol, S. Klingebiel, L. Pálfalvi, F. Krausz, S. Karsch, and J. Hebling, "Efficient generation of THz pulses with $0.4 \mathrm{~mJ}$ energy," Opt. Express 22, 20155 (2014).

38. S.-W. Huang, E. Granados, W. R. Huang, K.-H. Hong, L. E. Zapata, and F. X. Kartner, "High conversion efficiency, high energy terahertz pulses by optical rectification in cryogenically cooled lithium niobate," Opt. Lett. 38, 796 (2013)

39. M. I. Bakunov and S. B. Bodrov, "Terahertz generation with tilted-front laser pulses in a contact-grating scheme," J. Opt. Soc. Am. B 31, 2549 (2014).

40. J. A. Fulop, G. Polonyi, B. Monoszlai, G. Andriukaitis, T. Balciunas, A. Pugzlys, G. Arthur, A. Baltuska, and J. Hebling, "Highly efficient scalable monolithic semiconductor terahertz pulse source," Optica 3, 1075 (2016).

41. J. Hebling and Z. Marton, "Theory of spectroscopic devices," J. Opt. Soc. Am. A 23, 966 (2006)

42. X. Wu, S. Carbajo, K. Ravi, F. Ahr, G. Cirmi, Y. Zhou, O. D. Mücke, and F. X. Kartner, "Terahertz generation in lithium niobate driven by Ti:sapphire laser pulses and its limitations," Opt. Lett. 39, 5403 (2014).

43. S.-C. Zhong, Z.-H. Zhai, J. Li, L.-G. Zhu, J. Li, K. Meng, Q. Liu, L.-H. Du, J.-H. Zhao, and Z.-R. Li, "Optimization of terahertz generation from $\mathrm{LiNbO}_{3}$ under intense laser excitation with the effect of three-photon absorption," Opt. Express 23, 31313 (2015).

44. K. Ravi, B. K. Ofori-Okai, K. A. Nelson, and F. X. Kartner, "Analysis of terahertz generation by beamlet superposition," Opt. Express 27, 26547 (2019).

45. L. Wang, T. Kroh, N. H. Matlis, and F. Kaertner, "Full 3D+1 modeling of the tilted-pulse-front setups for single-cycle terahertz generation," J. Opt. Soc. Am. B 37, 1000 (2020).

46. Z. Chen, X. Zhou, C. A. Werley, and K. A. Nelson, "Generation of high power tunable multicycle teraherz pulses," Appl. Phys. Lett. 99, 071102 (2011).

47. F. Ahr, S. W. Jolly, N. H. Matlis, S. Carbajo, T. Kroh, K. Ravi, D. N. Schimpf, J. Schulte, H. Ishizuki, T. Taira, A. R. Maier, and F. X. Kartner, "Narrowband terahertz generation with chirped-and-delayed laser pulses in periodically poled lithium niobate," Opt. Lett. 42, 2118 (2017).

48. S. W. Jolly, N. H. Matlis, F. Ahr, V. Leroux, T. Eichner, A.-L. Calendron, H. Ishizuki, T. Taira, F. X. Kartner, and A. R. Maier, "Spectral phase control of interfering chirped pulses for high-energy narrowband terahertz generation," Nat. Commun. 10, 2591 (2019). 
49. G. Toth, J. A. Fulop, and J. Hebling, "Periodically intensity-modulated pulses by optical parametric amplification for multicycle tunable terahertz pulse generation," Opt. Express 25, 28258 (2017).

50. V. Stummer, T. Flory, G. Krizsan, G. Polonyi, E. Kaksis, A. Pugzlys, J. Hebling, J. A. Fulop, and A. Baltuska, "Programmable generation of terahertz bursts in chirped-pulse laser amplification," Optica 7, 1758 (2020).

51. L. Wang, G. Toth, J. Hebling, and F. Kartner, "Tilted-pulse-front schemes for terahertz generation,” Laser Photon. Rev. 14, 2000021 (2020).
52. G. Illes, Z. Tibai, L. Palfalvi, G. Almasi, J. Hebling, and G. Toth, "General characteristics of imaging-free nonlinear echelon slab to generate high energy $\mathrm{THz}$ pulses," in preparation.

53. J. A. Fulop, G. Polonyi, G. Krizsan, N. M. Mbithi, P. S. Nugraha, G. Almasi, L. Palfalvi, Z. Tibai, G. Toth, and J. Hebling, "Novel intense single- and multicycle THz sources," in 45th International Conference on Infrared, Millimeter, and Terahertz Waves (2020). 